Gut and Liver, Vol. 13 No. 4, July 2019, pp. 409-414

\title{
Gastric Xanthomas and Fundic Gland Polyps as Endoscopic Risk Indicators of Gastric Cancer
}

\author{
Kentaro Yamashita ${ }^{1}$, Ryo Suzuki ${ }^{1}$, Toshiyuki Kubo ${ }^{1}$, Kei Onodera $^{1}$, Tomoya lida $^{1}$, Mayuko Saito ${ }^{1}$, Yoshiaki Arimura ${ }^{2}$,
} Takao Endo ${ }^{3}$, Masanori Nojima ${ }^{4}$, and Hiroshi Nakase ${ }^{1}$

${ }^{1}$ Department of Gastroenterology and Hepatology, Sapporo Medical University, Sapporo, ${ }^{2}$ Department of Gastroenterology, Otaru General Hospital, Otaru, ${ }^{3}$ Department of Gastroenterology, Sapporo Shirakaba-dai Hospital, Sapporo, and ${ }^{4}$ Center for Translational Research, The Institute of Medical Science, The University of Tokyo, Tokyo, Japan

Background/Aims: Fundic gland polyps (FGPs), hyperplastic polyps (HPs), and xanthomas (XTs) are common benign gastric lesions that can be diagnosed by endoscopic appearance alone in most cases. The aim of this study was to evaluate associations between gastric cancer and these benign lesions. Methods: Two expert endoscopists reviewed a series of gastroscopy images. FGPs, HPs, and XTs were diagnosed by endoscopic appearance, whereas all gastric cancers were confirmed pathologically. Results: Of the 1,227 patients reviewed, 114 (9.3\%) had a concurrent or past history of gastric cancer. The overall prevalences of FGPs, HPs and XTs were $9.4 \%, 6.3 \%$ and $14.2 \%$, respectively. HPs and XTs coexisted in $1.6 \%$ of patients, whereas other combinations were rarer. XTs were observed in $39.3 \%$ and $11.5 \%$ of patients with and without gastric cancer, respectively $(p<0.001)$. In contrast, no gastric cancer patients had FGPs, whereas $10.4 \%$ of patients without cancer had FGPs $(p<0.001)$. The prevalence of HPs was similar between the two groups $(8.8 \%$ and $6.0 \%$ of patients with and without cancer, respectively, $p=0.29$ ). Multivariate and Mantel-Haenszel analyses demonstrated that XTs were positively associated and FGPs were negatively associated with gastric cancer. Conclusions: XTs and FGPs might be useful as endoscopic risk indicators for monitoring gastric cancer. (Gut Liver 2019;13:409-414)

Key Words: Fundic gland polyps; Gastric xanthomas; Hyperplastic polyps; Stomach neoplasms

\section{INTRODUCTION}

Helicobacter pylori infection is the strongest risk factor for gastric cancer, ${ }^{1}$ but $H$. pylori detection is frequently negative when gastric cancer is diagnosed., ${ }^{2,3}$ Atrophic gastritis and subsequent intestinal metaplasia (IM), mainly caused by $H$. pylori, are established endoscopic risk indicators of gastric cancer. ${ }^{4,5}$ However, endoscopic evaluations of atrophy and IM occasionally yield equivocal findings, making diagnosis difficult without biopsy. ${ }^{6,7}$ Magnifying and image-enhanced endoscopy might be useful for diagnosing atrophic gastritis, ${ }^{7}$ but it is time consuming and available at a limited number of facilities. Pathological confirmation of atrophic gastritis and IM for all patients is not practical in $H$. pylori endemic areas. Thus, endoscopic risk indicators other than atrophic gastritis are needed for gastric cancer surveillance.

Fundic gland polyps (FGPs), hyperplastic polyps (HPs), and xanthomas (XTs) are the most common benign lesions of the stomach. ${ }^{8,9}$ When these lesions demonstrate typical endoscopic findings, a diagnosis can be made without pathological confirmation. ${ }^{10}$ This "snap diagnosis" is possible for the majority of FGPs, HPs, and XTs. ${ }^{10}$ Past studies have demonstrated that HPs are positively correlated with $H$. pylori infection, ${ }^{10-12}$ whereas FGPs are negatively correlated with $H$. pylori infection. ${ }^{8,13}$ Fewer studies have been performed on XTs than on FGPs and HPs, but several reports have suggested that a positive correlation exists between XTs and $H$. pylori infection. ${ }^{9,14}$ Some studies have demonstrated that HPs are frequently concurrent with gastric cancer, ${ }^{15}$ but the correlation is still controversial. Thus, while correlations between $H$. pylori infection and these benign gastric lesions have been demonstrated, a direct association between these benign gastric lesions and gastric cancer has not been determined. Because most gastric cancers arise from background mucosa with current or past $H$. pylori infection, ${ }^{2,16}$ we hypothesized that the existence of these benign gastric lesions might positively or negatively correlate with gastric cancer. We there-

Correspondence to: Kentaro Yamashita

Department of Gastroenterology and Hepatology, Sapporo Medical University, S1W16 Chuo-ku, Sapporo 060-8543, Japan

Tel: +81-11-6112111, Fax: +81-11-6112282, E-mail: ykentaro@sapmed.ac.jp

Received on March 21, 2017. Revised on October 6, 2018. Accepted on October 18, 2018. Published online March 15, 2019

pISSN 1976-2283 eISSN 2005-1212 https://doi.org/10.5009/gnl17136

@ This is an Open Access article distributed under the terms of the Creative Commons Attribution Non-Commercial License (http://creativecommons.org/licenses/by-nc/4.0) which permits unrestricted non-commercial use, distribution, and reproduction in any medium, provided the original work is properly cited. 
fore investigated the prevalence of these benign gastric lesions and their association with gastric cancer to determine whether these benign lesions might be endoscopic risk indicators of gastric cancer.

\section{MATERIALS AND METHODS}

\section{Study design}

This was a retrospective study in which two board-certified expert endoscopists (K.Y. and R.S.) reviewed a series of upper gastrointestinal (GI) endoscopy images obtained at Sapporo Medical University Hospital from January to December 2010 and maintained on an electronic filing system (Solemio; Olympus, Tokyo, Japan). Panendoscopes (GIF-XQ260, GIF-Q260 or GIF-H260; Olympus Medical Systems, Tokyo, Japan) and an electronic endoscope system (EVIS LUCERA system, Olympus Medical Systems) were used for gastroscopy.

\section{Diagnostic definition}

Endoscopic diagnoses for the three types of benign lesions are defined as follows. FGPs are small (usually $<1 \mathrm{~cm}$ ) sessile polyps of the gastric body or fundus, with a smooth surface and normal coloration without erosion (Fig. 1A). ${ }^{10}$ Although HPs vary in size, are either sessile or pedunculated, and either have erosion on the surface or not, their most distinguishing feature is their red color (Fig. 1B). ${ }^{12}$ HPs can be observed at any site in the stomach. XTs are small (usually $<1 \mathrm{~cm}$ ) plaques with a rough surface and yellow-gray coloration (Fig. 1C). ${ }^{9,14}$ XTs are often observed in multiples and can be found at any site.

When endoscopic images demonstrated the typical findings mentioned above, FGPs, HPs and XTs were diagnosed from the endoscopic appearance without a biopsy. Lesions with atypical or equivocal appearances were included only when confirmed pathologically. Lesions with conflicting diagnoses by two reviewers were excluded. Patients with concurrent gastric cancer or a past history of gastric cancer who underwent endoscopic resection or partial gastrectomy at our hospital were regarded as gastric cancer patients. All gastric cancers were confirmed pathologically. Extensive atrophy was defined as the open-type atrophy of the Kimura-Takemoto classification. ${ }^{17}$

\section{Statistical analysis}

The mean ages of the patient groups were compared using the Student t-test. Associations between the prevalence of the three benign lesions and gastric cancer were assessed using the chi-square test. Logistic regression was used for multivariate analysis, and the Mantel-Haenszel test was used for matchedpair analysis. All p-values were two-sided, and the results were considered significant when $\mathrm{p}$-values were $<0.05$. We used SPSS version 20.0 (IBM Corp., Armonk, NY, USA) for statistical analysis. The Institutional Review Board of Sapporo Medical University approved this study (IRB number: 272-134). The informed consent was waived.

\section{RESULTS}

\section{Clinicopathological characteristics of the patients and gastric cancers}

In total, the endoscopic images of 1,227 Japanese patients who underwent upper GI endoscopy were reviewed. The mean age of the patients was 63.2 years (range, 15 to 92 years), and $48.3 \%$ of the patients were male. Of the 1,227 patients, 114 (9.3\%) had histologically confirmed gastric cancer. Fifty-eight patients had concurrent gastric cancer, whereas 56 had a past history of gastric cancer (Table 1). Of patients with a past history, 40 were treated with endoscopic resection, and 16 were treated with distal gastrectomy. Compared with the 1,113 patients without cancer, the gastric cancer patients were significantly older and predominantly male. The mean ages of gastric cancer patients and patients without cancer were 69.9 years (range, 37 to 89 years) and 62.3 years (range, 15 to 92 years), respectively $(\mathrm{p}<0.0001)$. The percentages of males among patients with and without gastric cancer were $79.8 \%$ and $45.1 \%$, respectively $(p<0.0001)$. The profiles of gastric cancers are sum-
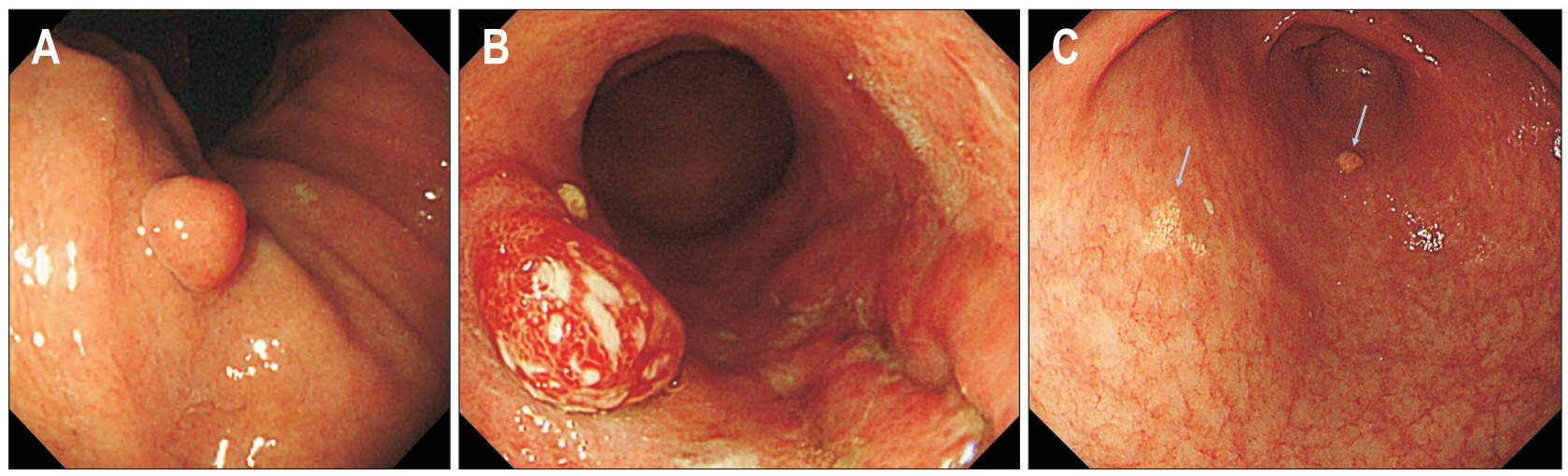

Fig. 1. (A) Endoscopic image of a fundic gland polyp. (B) Endoscopic image of a gastric hyperplastic polyp. (C) Endoscopic image of gastric xanthomas (arrows). 
marized in Table 1. One-hundred fourteen patients had a total of 127 gastric cancers, that is, 13 patients (11.4\%) had double gastric cancer. Ninety-seven cases (82.2\%) were at Union for International Cancer Control (UICC) stage I, 92\% of the lesions were located in the middle or lower stomach, and 71.7\% of the lesions could be categorized as Lauren intestinal subtype. Among the gastric cancer patients, extensive atrophic gastritis

Table 1. Characteristics of Gastric Cancer

\begin{tabular}{lc}
\hline \multicolumn{1}{c}{ Characteristic } & No. (\%) \\
\hline UICC stage & $97(82.2)$ \\
I & $6(5.0)$ \\
II & $4(3.4)$ \\
III & $11(9.3)$ \\
IV & \\
Site & $13(10.2)$ \\
Upper & $80(63.0)$ \\
Middle & $33(26.0)$ \\
Lower & $1(0.8)$ \\
Whole & \\
Histology & $91(71.7)$ \\
Intestinal & $26(20.5)$ \\
Diffuse & $10(7.9)$ \\
Mixed & $13(11.4)$ \\
Double gastric cancers & $58(50.9)$ \\
Concurrent gastric cancer & \\
Past gastric cancer & $40(35.1)$ \\
Endoscopic resection & $16(14.0)$ \\
Distal gastrectomy & $105(92.1)$ \\
Extensive atrophy & \\
Serum $H$. pylori antibody & $114 / 127$ \\
Positive & $p y 1.6)$ \\
Notal, no. of patients/cancers & \\
\hline & \\
\hline & \\
\hline
\end{tabular}

UICC, Union for International Cancer Control; H. pylori, Helicobacter pylori. was observed in 92.1\% (105/114), and serum samples were positive for the anti-H. pylori antibody in 52\% (33/64).

\section{Prevalences of three benign gastric lesions and their as- sociations with gastric cancer}

The two experts made identical endoscopic diagnoses in 366 of 375 (97.6\%) benign gastric lesions, and the nine lesions with conflicting diagnoses were excluded from the analysis. The overall prevalences of FGPs, HPs, and XTs were 9.4\%, 6.3\%, and 14.2\%, respectively (Table 2). The coexistence of HPs and XTs was observed in $1.6 \%$ of patients, whereas combinations of FGPs and the other two lesions were rarer. No patient had all three benign gastric lesions simultaneously.

Next, the prevalences of the three benign gastric lesions were compared between patients with and without gastric cancer (Table 2). FGPs were observed in $10.4 \%$ of patients without gastric cancer, whereas no gastric cancer patients had FGPs ( $p=0.0002)$. In contrast, XTs were observed in $40.4 \%$ and $11.5 \%$ of patients with and without gastric cancer, respectively $(\mathrm{p}<0.0001)$. Gastric cancer with XTs were predominantly intestinal-type cancers compared with those without XTs (93.5\% vs 67.8\%, $\mathrm{p}=0.003$ ), whereas no differences were observed between the two groups in age, sex, site, concurrent or past history of gastrectomy, or stage (Table 3). The was no difference in the prevalence of HPs between the two groups, although a slightly higher rate was observed in gastric cancer patients $(8.8 \%$ and $6.0 \%$ in patients with and without cancer, respectively, $\mathrm{p}=0.29$ ).

Multivariate analysis demonstrated that the presence of XTs was an independent risk factor for gastric cancer (Table 4). FGPs could not be analyzed by multivariate analysis because the prevalence of FGPs in gastric cancer patients was zero. We therefore performed a Mantel-Haenszel analysis, which showed that the presence of FGPs was inversely correlated with gastric cancer (Table 4).

\section{DISCUSSION}

To the best of our knowledge, this is the first report on correlations between the three common benign gastric lesions and

Table 2. Prevalences and Associations between Three Benign Lesions and Gastric Cancer

\begin{tabular}{lcccc}
\hline Benign gastric lesions & Total $(\mathrm{n}=1,227)$ & Gastric cancer patients $(\mathrm{n}=114)$ & Patients without cancer $(\mathrm{n}=1,113)$ & $\mathrm{p}$-value \\
\hline FGPs & $115(9.4)$ & 0 & $115(10.4)$ & $67(6.0)$ \\
HPs & $77(6.3)$ & $10(8.8)$ & $128(11.5)$ & 0.0002 \\
XTs & $174(14.2)$ & $46(40.4)$ & $3(0.3)$ & -29 \\
FGPs+HPs & $3(0.2)$ & 0 & $1(0.1)$ & - \\
FGPs+XTs & $1(0.1)$ & 0 & $15(1.3)$ & - \\
HPs+XTs & $20(1.6)$ & $5(4.4)$ & - & - \\
FGPs+HPs+XTs & 0 & - & -
\end{tabular}

Data are presented as number $(\%)$.

FGPs, fundic gland polyps; HPs, hyperplastic polyps; XTs, gastric xanthomas. 
gastric cancer. First, XTs are one of the most common benign gastric lesions, but the etiology and clinical significance of XTs remain unknown. Pathologically, XTs were composed of foamy histiocytes containing lipids aggregated in the lamina propria (Fig 2). Several studies have demonstrated close correlations among XTs, H. pylori infection and atrophic gastritis. ${ }^{9,14}$ Hori and Tsutsumi ${ }^{14}$ suggested that $H$. pylori invasion in the lamina propria was an etiology of XTs by showing that $H$. pylori antigens existed in the cytoplasm of XT cells. Isomoto et al. ${ }^{9}$ also demonstrated that the $H$. pylori infection rate was significantly higher in patients with XTs than in age- and sex-matched controls without XTs. In this study, patients with XTs had more extensive atrophic gastritis than patients in the control group, and $H$. pylori antigens were identified in the XT cells of $86 \%$ of $H$. pylori-positive patients with XTs. The close association between XTs and gastric cancer in our study might indicate extensive atrophic gastritis of the background mucosa. Because XTs have no malignant potential per se, endoscopists often omit describing the presence of XTs in reports. However, retrospective and prospective studies demonstrating a correlation between XTs and early gastric cancer have recently been published. ${ }^{18,19}$ Our study also suggests that XTs might be useful as an endoscopic risk indicator of gastric cancer. Future studies are necessary to clarify whether XTs are risk indicators of gastric cancer in patients with extensive atrophy.

Second, HPs are thought to be a consequence of the mucosal regenerative response to chronic inflammation. The etiology of

Table 3. Comparison of Gastric Cancer with and without Xanthomas

\begin{tabular}{lccc}
\hline Clinical parameters & $\begin{array}{c}\text { Cancer with XTs } \\
(\mathrm{n}=46)\end{array}$ & $\begin{array}{c}\text { Cancer without XTs } \\
(\mathrm{n}=68)\end{array}$ & $\mathrm{p}$-value \\
\hline Mean age (yr) & 70.9 & 69.2 & 0.38 \\
Male sex & $38(82.6)$ & $53(77.9)$ & 0.54 \\
U-region & $6(13.0)$ & $7(10.3)$ & 0.65 \\
Intestinal type & $40(87.0)$ & $38(55.9)$ & 0.0004 \\
Concurrent cancer & $22(47.8)$ & $36(52.9)$ & 0.59 \\
Past gastrectomy & $4(8.7)$ & $12(17.6)$ & 0.17 \\
\hline
\end{tabular}

Data are presented as mean or number (\%).

XTs, gastric xanthomas.
HPs includes peptic ulcers, gastroenteric anastomosis, autoimmune gastritis and $H$. pylori gastritis. ${ }^{10,15}$ Eighty percent of HPs regressed after the eradication of $H$. pylori in a prospective study, ${ }^{11}$ and other studies demonstrated that HPs were an endoscopic risk indicator of gastric cancer. ${ }^{10,15}$ In contrast, our study found no association between HPs and gastric cancer, although the prevalence of HPs was higher in patients with gastric cancer. The reason for this discrepancy is not clear, but it might be because our study included more HP cases not associated with $H$. pylori gastritis than previous studies.

Third, FGPs are the most common gastric polyps in developed countries, particularly in areas with low $H$. pylori infection rates. $^{8,10}$ The worldwide prevalence of FGPs is likely increasing, probably due to the increased long-term use of proton pump inhibitors (PPIs) and decreases in the $H$. pylori infection rates. ${ }^{8,10}$ Whether long-term PPI use is associated with the development of FGPs remains controversial, ${ }^{20,21}$ but $H$. pylori infection is thought to be a protective factor against FGPs. Carmack et al. ${ }^{8}$ analyzed a United States database and reported that the rate of $H$. pylori infection among patients with FGPs was 0.1\% (6 of $6,065)$, significantly lower than the $14.0 \%(9,939$ of 71,172$)$ that was reported for patients without any gastric polyps (hazard ratio, 0.007 ; 95\% confidence interval, 0.003 to 0.0016). In our study, FGPs were found in $10.4 \%$ of the patients without gastric

Table 4. Multivariate Analysis and Mantel-Haenszel Analysis of Factors Predicting Gastric Cancer

\begin{tabular}{llccr}
\hline \multicolumn{2}{c}{ Statistical methods } & OR & $95 \%$ CI & p-value \\
\hline Multivariate analysis & Age & 1.05 & $1.02-1.07$ & $<0.001$ \\
& Female & 0.24 & $0.15-0.39$ & $<0.001$ \\
& HPs & 1.40 & $0.65-3.00$ & 0.392 \\
& XTs & 3.33 & $2.14-5.17$ & $<0.001$ \\
Mantel-Haenszel analysis & FGPs & NA & NA & 0.006 \\
& HPs & 1.85 & $0.89-3.85$ & 0.147 \\
& XTs & 3.68 & $2.38-5.70$ & $<0.001$ \\
\hline
\end{tabular}

Multivariate analysis was not suitable for fundic gland polyps (FGPs) because FGPs were never observed in gastric cancer patients. OR, odds ratio; CI, confidence interval; HPs, hyperplastic polyps; XTs, gastric xanthomas; NA, not available.
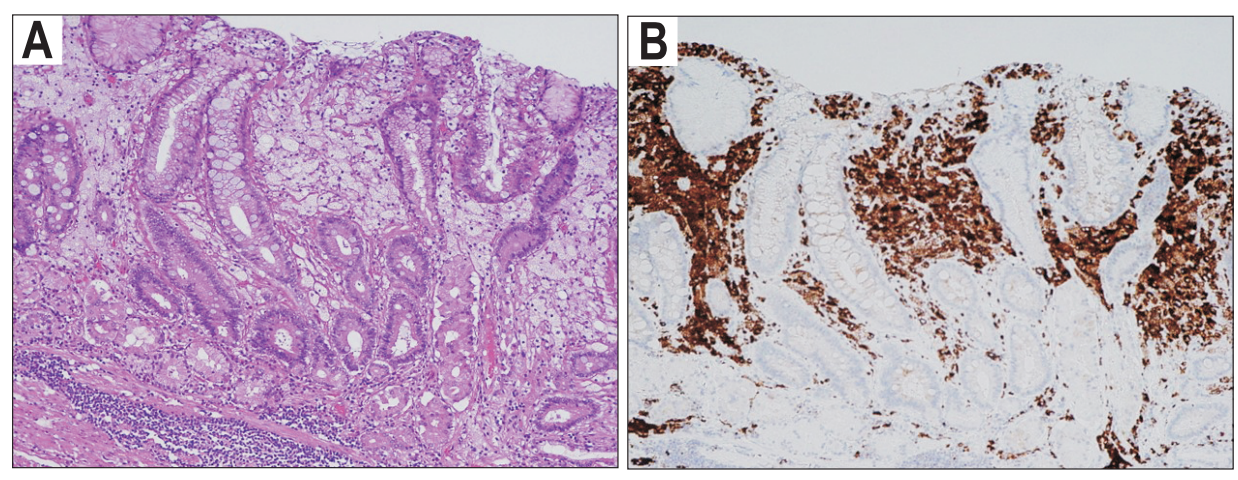

Fig. 2. (A) Pathological findings of an xanthoma (XT; H\&E stain, $\times 100$ ). (B) Immunohistochemistry for CD68 (KP1), a marker of macrophages, in an XT $(\times 100)$. 
cancer, while none of the 114 gastric cancer patients had FGPs. Additionally, in a study from the United States and Puerto Rico, gastric cancer was diagnosed in 0 out of 6,081 patients with FGPs and in $0.14 \%$ (178 of 72,797) of patients without FGPs. ${ }^{13}$ These negative correlations between FGPs and gastric cancer are probably due to the protective effect of $H$. pylori infection against the development of FGPs. Therefore, FGPs might be a useful indicator of a low risk of developing gastric cancer.

The limitations of our study were as follows: (1) the study design was retrospective; (2) lesions difficult to diagnose by imaging were excluded; (3) half of the gastric cancer patients had received treatment in the past, and 14\% of the cancer patients had undergone distal gastrectomy, which can cause bile reflux and subsequent chronic inflammation; and (4) there was a lack of data regarding the current $H$. pylori status, eradication history, degree of atrophy, and medications, including PPIs. These factors might have influenced the prevalences of the three benign gastric lesions. A prospective study to clarify the best endoscopic predictive marker for gastric cancer among XTs, FGPs, atrophic gastritis and IM is necessary in the future.

In conclusion, our study demonstrates that XTs and FGPs are positively and negatively associated with a concurrent or past history of gastric cancer, respectively. XTs and FGPs might be useful predictive endoscopic markers for monitoring gastric cancer.

\section{CONFLICTS OF INTEREST}

No potential conflict of interest relevant to this article was reported.

\section{ORCID}

Kentaro Yamashita

Ryo Suzuki

Toshiyuki Kubo

Tomoya Iida

Yoshiaki Arimura

Takao Endo

Masanori Nojima

Hiroshi Nakase https://orcid.org/0000-0001-9003-9846 https://orcid.org/0000-0002-5339-2429 https://orcid.org/0000-0002-0892-8298 https://orcid.org/0000-0003-2842-8052 https://orcid.org/0000-0002-3156-1500 https://orcid.org/0000-0001-7808-111X https://orcid.org/0000-0002-8788-2522 https://orcid.org/0000-0003-2848-6586

\section{REFERENCES}

1. Infection with Helicobacter pylori. IARC Monogr Eval Carcinog Risks Hum 1994;61:177-240.

2. Kato S, Matsukura N, Tsukada K, et al. Helicobacter pylori infection-negative gastric cancer in Japanese hospital patients: incidence and pathological characteristics. Cancer Sci 2007;98:790794.

3. Kang HY, Kim N, Park YS, et al. Progression of atrophic gastritis and intestinal metaplasia drives Helicobacter pylori out of the gas- tric mucosa. Dig Dis Sci 2006;51:2310-2315.

4. Ohata H, Kitauchi S, Yoshimura N, et al. Progression of chronic atrophic gastritis associated with Helicobacter pylori infection increases risk of gastric cancer. Int J Cancer 2004;109:138-143.

5. Lahner E, Bordi C, Cattaruzza MS, et al. Long-term follow-up in atrophic body gastritis patients: atrophy and intestinal metaplasia are persistent lesions irrespective of Helicobacter pylori infection. Aliment Pharmacol Ther 2005;22:471-481.

6. Redéen S, Petersson F, Jönsson KA, Borch K. Relationship of gastroscopic features to histological findings in gastritis and Helicobacter pylori infection in a general population sample. Endoscopy 2003;35:946-950.

7. Gonen C, Simsek I, Sarioglu S, Akpinar H. Comparison of high resolution magnifying endoscopy and standard videoendoscopy for the diagnosis of Helicobacter pylori gastritis in routine clinical practice: a prospective study. Helicobacter 2009;14:12-21.

8. Carmack SW, Genta RM, Schuler CM, Saboorian MH. The current spectrum of gastric polyps: a 1-year national study of over 120,000 patients. Am J Gastroenterol 2009;104:1524-1532.

9. Isomoto H, Mizuta Y, Inoue K, et al. A close relationship between Helicobacter pylori infection and gastric xanthoma. Scand J Gastroenterol 1999;34:346-352.

10. Goddard AF, Badreldin R, Pritchard DM, Walker MM, Warren B; British Society of Gastroenterology. The management of gastric polyps. Gut 2010;59:1270-1276.

11. Ohkusa T, Takashimizu I, Fujiki K, et al. Disappearance of hyperplastic polyps in the stomach after eradication of Helicobacter pylori: a randomized, clinical trial. Ann Intern Med 1998;129:712715.

12. Jain R, Chetty R. Gastric hyperplastic polyps: a review. Dig Dis Sci 2009;54:1839-1846.

13. Genta RM, Schuler CM, Robiou CI, Lash RH. No association between gastric fundic gland polyps and gastrointestinal neoplasia in a study of over 100,000 patients. Clin Gastroenterol Hepatol 2009;7:849-854.

14. Hori S, Tsutsumi Y. Helicobacter pylori infection in gastric xanthomas: immunohistochemical analysis of 145 lesions. Pathol Int 1996;46:589-593.

15. Dirschmid K, Platz-Baudin C, Stolte M. Why is the hyperplastic polyp a marker for the precancerous condition of the gastric mucosa? Virchows Arch 2006;448:80-84.

16. Matsuo T, Ito M, Takata S, Tanaka S, Yoshihara M, Chayama K. Low prevalence of Helicobacter pylori-negative gastric cancer among Japanese. Helicobacter 2011;16:415-419.

17. Kimura K, Takemoto T. An endoscopic recognition of the atrophic border and its significance in chronic gastritis. Endoscopy 1969;1:87-97.

18. Sekikawa A, Fukui H, Maruo T, et al. Gastric xanthelasma may be a warning sign for the presence of early gastric cancer. J Gastroenterol Hepatol 2014;29:951-956.

19. Sekikawa A, Fukui H, Sada R, et al. Gastric atrophy and xanthelasma are markers for predicting the development of early gastric 
414 Gut and Liver, Vol. 13, No. 4, July 2019

cancer. J Gastroenterol 2016;51:35-42.

20. Jalving M, Koornstra JJ, Wesseling J, Boezen HM, DE Jong S, Kleibeuker JH. Increased risk of fundic gland polyps during longterm proton pump inhibitor therapy. Aliment Pharmacol Ther
2006;24:1341-1348.

21. Vieth M, Stolte M. Fundic gland polyps are not induced by proton pump inhibitor therapy. Am J Clin Pathol 2001;116:716-720. 